\title{
ORIGINAL
}

\section{INFLUENCIA DE LA ESTRUCTURA DE LA POBLACIÓN EN EL CONSUMO DE ANTIBIÓTICOS SISTÉMICOS EN LA PROVINCIA DE VALLADOLID}

Enrique Pastor García (1), José María Eiros Bouza (2) y Agustín Mayo Iscar (3)

(1) Centro de Salud Rondilla I. Valladolid

(2) Departamento de Microbiología. Hospital Clínico. Valladolid

(3) Departamento de Bioestadística. Facultad de Medicina. Valladolid

\section{RESUMEN}

Fundamento: El elevado consumo de antibióticos sistémicos en nuestro país hace necesario el estudio de aquellos factores que determinen su distribución. Diversos parámetros están implicados en el reparto desigual de su consumo, entre ellos: a) la epidemiología de los procesos infecciosos, b) los factores dependientes de la población y c) factores dependientes de los médicos prescriptores. En el presente trabajo pretendemos realizar un análisis de aquellos determinantes dependientes de la población (tipo de población y edad).

Métodos: Estudio longitudinal retrospectivo con datos de consumo de antibióticos de uso sistémico proporcionados por la compañía International Marketing Services (IMS) entre el 1 de enero de 1996 y el 31 de diciembre del 2000. El indicador de consumo utilizado fue el número de dosis diaria definida por 1000 habitantes y día (DHD). Se consideraron seis áreas geográficas en la provincia de Valladolid, tres de ellas de carácter urbano y otras tres predominantemente rurales.

Resultados: El consumo global por área fue el siguiente: Medina del Campo (25,9DHD), Valladolid capital (23,4DHD), Laguna de Duero (22,6DHD), Área Norte (22,4DHD), Área Sur $(21,4 \mathrm{DHD})$ y en último lugar el Área Centro (20,2DHD). El consumo específico por áreas determinó un mayor consumo de amoxicilina en las tres áreas urbanas, amoxicilina-clavulánico y los principales macrólidos en Medina del Campo, quinolonas en el Área Norte y tetraciclinas y sulfamidas en Valladolid capital

Conclusiones: Observamos diferencias globales de consumo importantes entre áreas, con unos máximos en las áreas urbanas. Estas diferencias fueron más marcadas al estudiar la distribución geográfica del consumo de los principales principios activos.

Palabras clave: Antibióticos. Medidas epidemiológicas. Demografía

Correspondencia

Enrique Pastor García

Centro de Salud Rondilla I.

C/ Cardenal Torquemada, 54

47010 - Valladolid

Teléfono: 983310155

Fax: 983310102

Correo electrónico: med007242@saludalia.com

\section{ABSTRACT}

\section{The Influence of Population Structure on Systemic Antibiotic Consumption Valladolid, Spain}

Background: The high degree to which systemic antibiotics are consumed in our country makes a study of those factors determining the spread thereof necessary. Different parameters are involved in the unequal spread of their consumption, including: a) the epidemiology of the infectious processes b) the population-dependent factors and c) factors depending upon the prescribing physicians. This study is aimed at analyzing those determining factors depending upon the population (type of population and age).

Methods: A longitudinal retrospective study based on systemic antibiotic consumption data provided by the company International Marketing Services (IMS) for the January 1, 1996-December 31, 2000 period. The consumption indicator used was the number of daily doses defined per 1000 inhabitants/day (DID). Six geographical areas in the province of Valladolid were placed under study, three of which were urban and the other three mainly rural.

Results: The overall consumption by area was as follows: Medina del Campo (25.9DID), the capital city of Valladolid (23.4DID), Laguna de Duero (22.6DID), Northern Area (22.4DID), Southern Area (21.4DID) and, lastly, the Central Area (20.2DID). The specific consumption by areas revealed a greater consumption of amoxicillin in the three urban areas, amoxicillin-clavulanic acid and the major macrolides in Medina del Campo, quinolone antibiotics in the Northern Area and tetracyclines and sulfonamides in the capital city of Valladolid.

Conclusions: Major overall consumption-related differences were found to exist among areas, the maximums being found in the urban areas. These differences were more marked on when studying the geographic spread of the consumption of the main active ingredients.

Keywords: Antibiotics. Epidemiologic measurements. Demography. 


\section{INTRODUCCIÓN}

El uso y consumo de antibióticos sistémicos está influenciado por múltiples parámetros, siendo unos más conocidos que otros. Entre los primeros estarían los distintos procesos infecciosos susceptibles de tratamiento con estos medicamentos y por ende los factores epidemiológicos que los caracteri$z^{2}{ }^{1}$, y que afectan tanto a la distribución de los distintos microorganismos como la diversificación de la población.

Otros factores menos conocidos que afectan a este consumo son la estructura demográfica de la población, tanto en su distribución por edades como por el tipo de población (urbana o rural), así como los factores dependientes de los médicos prescriptores (formación, lugar de trabajo, experiencia personal,...).

Existen numerosos estudios nacionales e internacionales que muestran un uso desmedido de este grupo de fármacos en nuestro país ${ }^{2,3}$ siendo por tanto necesarios nuevos trabajos epidemiológicos de uso y consumo que postulen patrones anómalos susceptibles de corrección, haciendo especial hincapié en aquellos factores menos conocidos de su distribución.

\section{MATERIAL Y MÉTODOS}

El presente estudio se llevó a cabo en la provincia de Valladolid, con datos de consumo de antibióticos sistémicos desde el primero de enero de 1.996 hasta el 31 de diciembre del 2000. Los datos poblacionales proceden del padrón municipal de $1.996^{4}$, al ser este una relación de residentes y transeúntes, esto es, tanto de los que viven habitualmente como de los que se encuentran accidentalmente en una zona. Son, por tanto, el grupo de población que potencialmente puede consumir antibióticos.
La información de consumo utilizada en el estudio procede de los datos proporcionados por IMS (International Marketing Services), que analiza el consumo de fármacos a partir de su salida desde el mayorista a las farmacias, no recogiendo de esta forma el consumo hospitalario. Hay que tener en cuenta que el $90 \%$ d.el consumo de antibióticos se realiza en Atención Primaria y que IMS sí contempla las ventas por prescripciones en consultas privadas y por automedicación, los datos proporcionados alcanzan prácticamente el 95\% del consumo.

En el presente estudio se analizó todo el consumo de antibacterianos sistémicos que se incluyen en la clasificación de la Anatomical Therapeutical Chemical Classification Index (ATC) del grupo J01: antibióticos de uso sistémico. No se incluyeron por tanto otros antiinfecciosos distintos de este grupo ni preparados tópicos dermatológicos, oftalmológicos o de la esfera ORL en cuya composición formen parte antibióticos de este grupo.

La información fue tabulada teniendo en cuenta el número de paquetes que cada marca vende cada año, y obteniendo los datos de consumo de acuerdo a la Dosis Diaria Definida (DDD).

El indicador de consumo utilizado es el número de DDD por 1000 habitantes y día (DHD), según la siguiente fórmula ${ }^{5}$ :

$$
\mathrm{DHD}=\frac{\mathrm{UV} \times \mathrm{FF} / \mathrm{E} \times \mathrm{C} / \mathrm{FF} \times 1.000}{\mathrm{DDD} \times \mathrm{N} .^{\circ} \text { de habitantes } \times 365 \text { días }}
$$

Siendo UV las unidades vendidas, FF/E el número de formas farmacéuticas por envase y $\mathrm{C} / \mathrm{FF}$ la cantidad de producto en cada forma farmacéutica.

Para el procesamiento de los datos se construyeron bases de datos en el programa Excel y Access. El análisis de los datos se hizo utilizando el paquete estadístico SAS (North Caroline). Los resultados del análi- 
sis se presentan según los indicadores de consumo en tablas y figuras.

\section{RESULTADOS}

En primer lugar presentamos el estudio de la población de la provincia de Valladolid, que muestra una variabilidad de acuerdo a las dos características estudiadas. Por un lado observamos tres núcleos urbanos (Valladolid capital, Medina de Campo y Laguna de Duero), siendo el resto de la provincia predominantemente de carácter rural, habiéndola dividido en tres zonas que por su situación geográfica hemos denominado áreas Norte, Centro y Sur. Por lo que respecta a la distribución por edades e índices en dichas áreas, hemos podido observar los siguientes datos (tabla 1):

- La mayor población pediátrica correspondía a Laguna de Duero. Asimismo, en esta zona, el índice de dependencia de $\geq 65$ años era tan sólo de un 5,1\%.

- El mayor índice de dependencia de $\geq 65$ años estaba situado en el Área Norte, seguido del Área Sur, siendo por tanto las zonas de mayor envejecimiento de la población de la provincia de Valladolid.

- Valladolid, Medina del Campo y el Área Centro presentaron unos índices equilibrados entre la población de $\leq 14$ años y $\operatorname{los} \geq 65$ años.

En segundo lugar mostramos los datos de consumo global por área geográfica, que están representados en la figura 1, donde observamos como el mayor consumo de antibióticos de uso sistémico correspondió a Medina del Campo con 25,9 DHD, seguido de Valladolid capital (23,4 DHD), Laguna de Duero (22,6 DHD), Área Norte (22,4 DHD), Ârea Sur (21,4 DHD) y en último lugar el Área Centro (20,2 DHD). Es interesante apuntar la diferencia entre el primero y el último, de casi 6 DHD.

Para apuntar posibles diferencias cualitativas de consumo realizamos un análisis de los principios activos que tenían un uso porcentual importante, representando dicha distribución en las figuras 2 a 4, donde observamos contrastes manifiestos:

En la figura 2 analizamos comparativamente el consumo de betalactámicos, obser-

Tabla 1

Índices de dependencia porcentual de la población de Valladolid

\begin{tabular}{|lcc|}
\hline \multicolumn{1}{|c|}{ Área } & Ind. Dep. $\leq 14$ & Ind. Dep. $\geq 65$ \\
\hline Valladolid & 13,7 & 13,8 \\
Laguna de Duero & 20,7 & 5,1 \\
Medina del Campo & 16,7 & 15,1 \\
Área Norte & 12,6 & 26,5 \\
Área Centro & 14,6 & 17,7 \\
Área Sur & 13,9 & 21,8 \\
\hline Media & $\mathbf{1 4 , 1}$ & $\mathbf{1 5 , 3}$ \\
\hline
\end{tabular}

\begin{tabular}{|l|}
\hline Índice de dependencia $\leq 14$ años $=\frac{\text { Población } \leq 14 \text { años }}{\text { Población total }} \times 100$ \\
\hline Índice de dependencia $\geq 65$ años $=\frac{\text { Población } \geq 65 \text { años }}{\text { Población total }} \times 100$ \\
\hline
\end{tabular}




\section{Figura 1}

Consumo medio anual de antibióticos de uso sistémico en DDD/1000hab/día por área geográfica

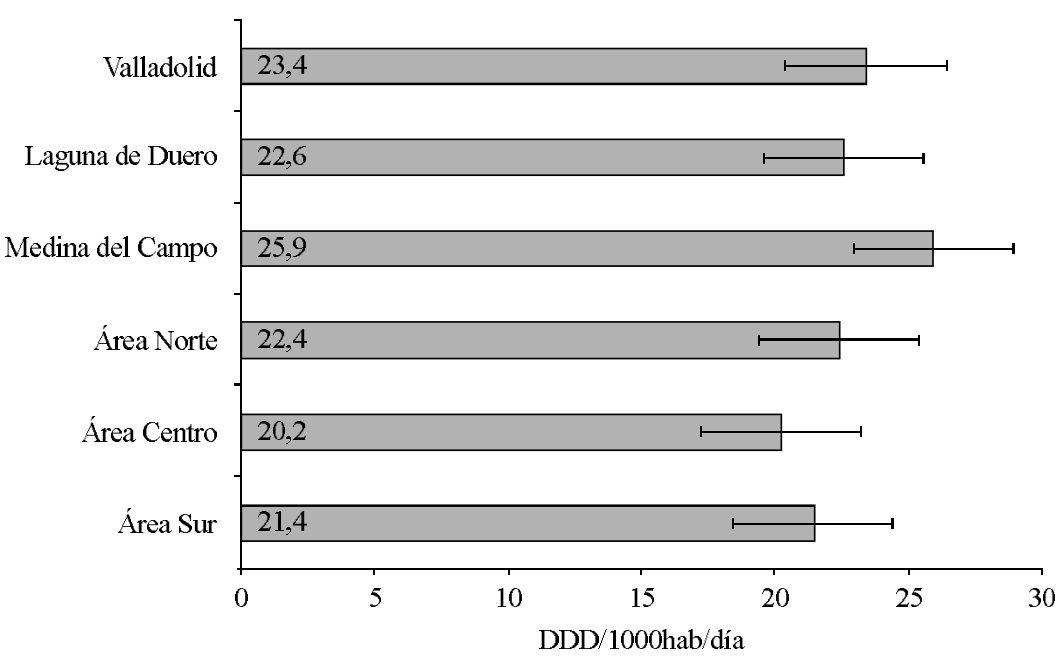

Figura 2

Consumo de los principales betalactámicos en DDD/1000hab/día por áreas

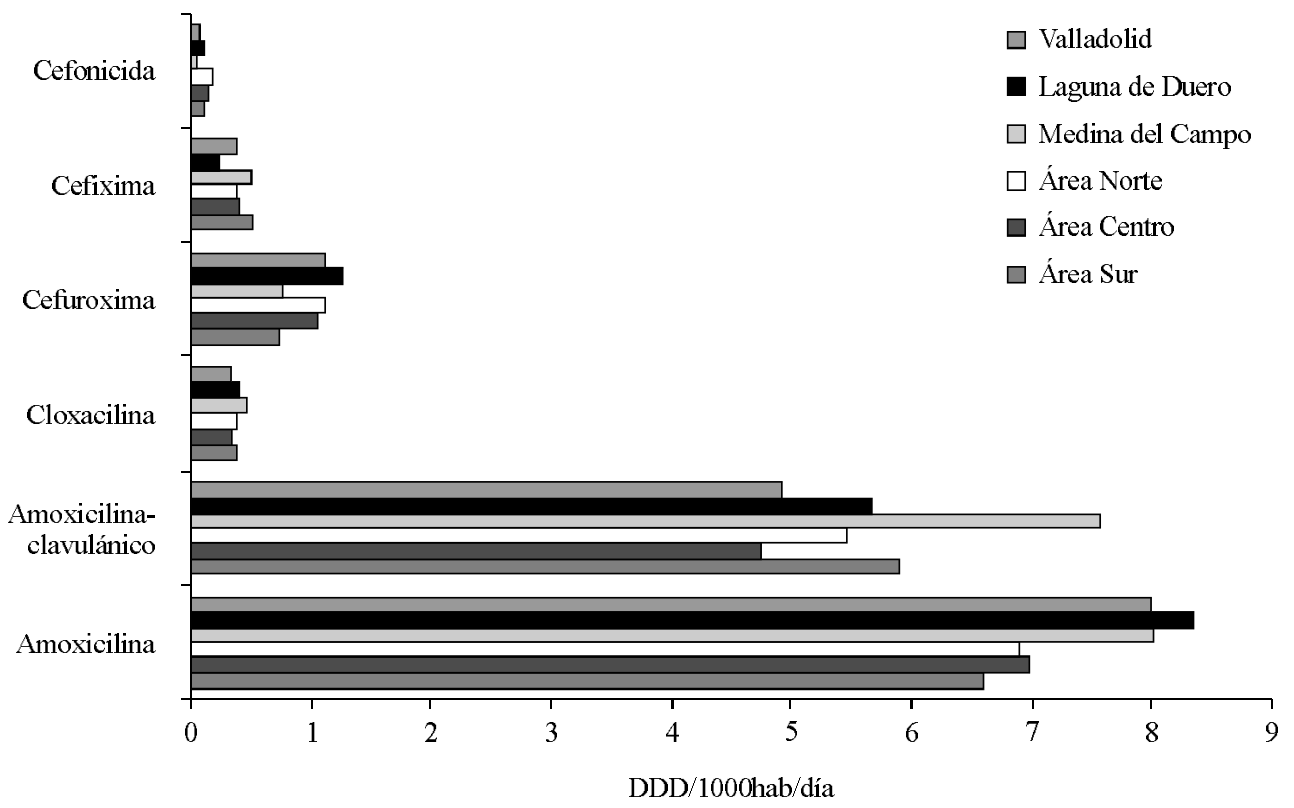


vando los siguientes hechos: la amoxicilina presentó un pico de consumo en Laguna de Duero (8,3 DHD) seguido de Valladolid capital y Medina del Campo (8,0 DHD). La asociación amoxicilina-clavulánico mostró grandes diferencias de consumo, siendo máximo en Medina del Campo (7,6 DHD) y mínimo en al Área Centro (4,7 DHD). El consumo de cloxacilina no mostró grandes diferencias entre áreas, oscilando entre 0,3 DHD en Valladolid capital y 0,5 DHD en Medina del Campo. Las cefalosporinas, con bajo consumo en todas las áreas, presentaron los siguientes datos máximos: cefuroxima en Laguna de Duero (1,3 DHD), cefixima en el Ârea Sur 0,5 DHD y cefonicida en el Área Norte (0,2 DHD).

Las diferencias por áreas fueron mayores en el subgrupo de los macrólidos que en los betalactámicos (Fig. 3): el consumo de claritromicina en Medina del Campo fue doble al originado en el Área Centro (1,5 y 0,7
DHD respectivamente). Las cifras de consumo de eritromicina mostraron una relación paralela a la claritromicina, con cifras entre 1,0 DHD en Medina del Campo y 0,4 DHD en el Área Centro. También fue Medina del Campo la que presentó mayor consumo de espiramicina (0,9 DHD), siendo menor en las áreas Norte y Centro (0,5 DHD). Por otra parte el consumo de midecamicina varió entre 0,6 DHD (Valladolid capital) y 0,3 DHD (Laguna de Duero). La azitromicina y la roxitromicina mostraron un gradiente de consumo según un patrón geográfico: la primera de Norte a Sur $(0,6$ a 0,3 DHD) y la segunda de Sur a Norte (también 0,6 a 0,3 DHD), aunque en este caso el menor uso correspondió a Laguna de Duero.

En la figura 4 hemos representado el resto de principios activos de los antibióticos de uso sistémico con un consumo importante, destacando los siguientes aspectos: el ciprofloxacino presentó un menor uso en Laguna

Figura 3

Consumo de los principales macrólidos en DDD/1000hab/día por áreas.

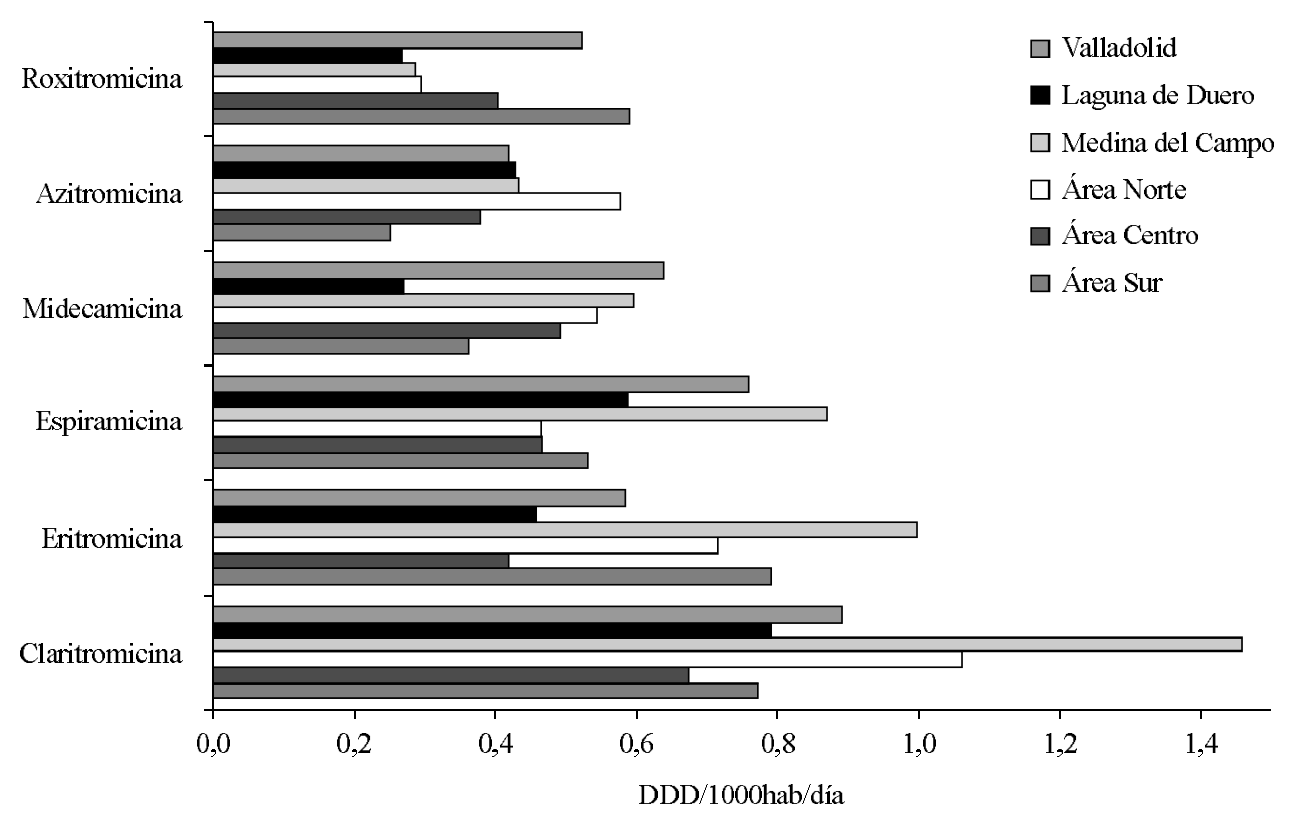


Figura 4

Consumo de los principales quinolonas, tetraciclinas y sulfamidas en DDD por 1000 habitantes y día por áreas

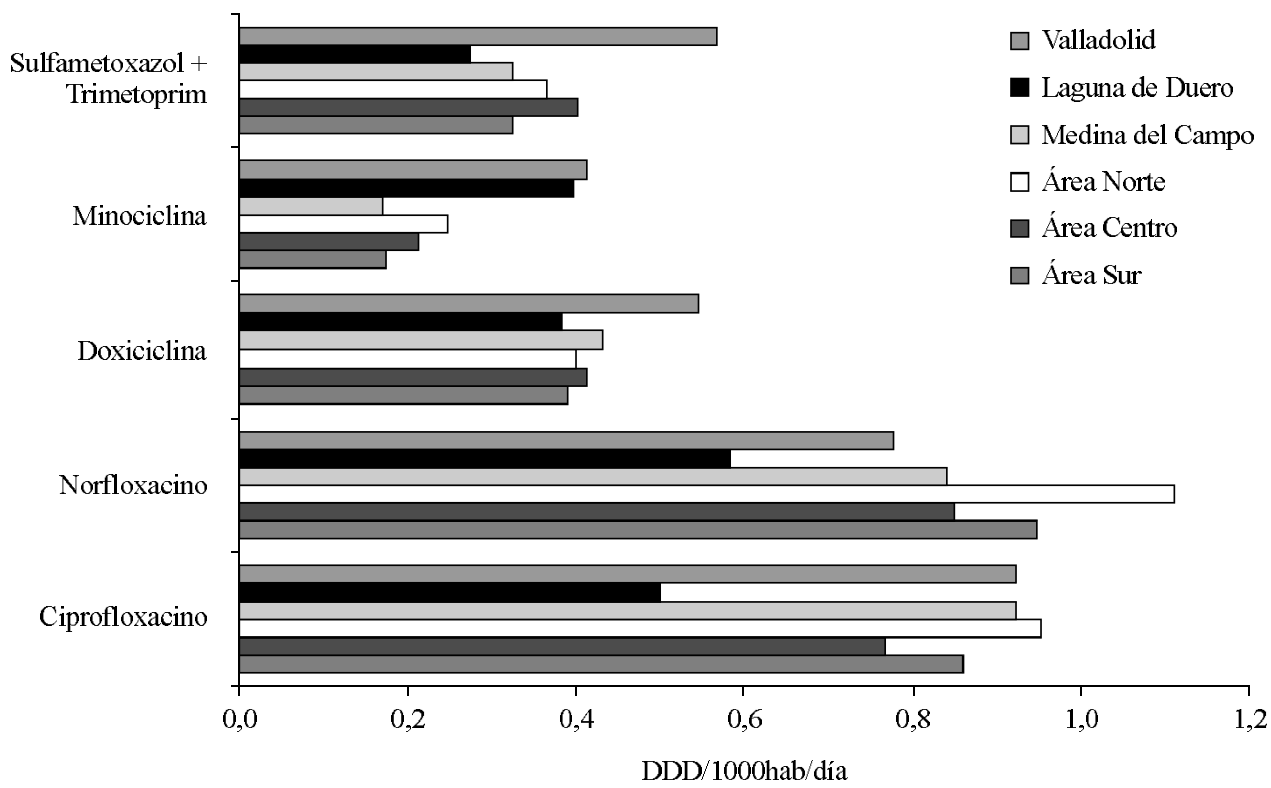

de Duero (0,5 DHD), mientras que en el resto de las áreas presentó un consumo en torno a las 0,9 DHD. También en Laguna de Duero el consumo de norfloxacino presentó el menor valor (0,6 DHD), siendo máximo en el Área Norte (1,1 DHD). En cuanto a las tetraciclinas, tanto la doxiciclina como la minociclina presentaron su valor máximo en Valladolid capital (0,5 DHD y 0,3 DHD respectivamente). Por último señalar que el consumo de sulfametoxazol + trimetoprim fue máximo en Valladolid capital $(0,6$ DHD) habiendo doblado el consumo respecto a Laguna de Duero (0,3 DHD).

\section{DISCUSIÓN}

Existen diversas circunstancias que pueden influir en el consumo diferencial de antibióticos entre regiones:

1. La estructura demográfica es sin duda uno de los factores más importantes que intervienen en el consumo de antibióticos en un área determinada ${ }^{6}$, de forma que la población pediátrica y los ancianos son potencialmente mayores consumidores de antibióticos.

2. La epidemiología de las infecciones, estando las zonas frías expuestas a una mayor tendencia hacia las infecciones respiratorias según demuestra la casuística de la mayoría de estos procesos.

3. Según datos aportados por los médicos en el «Estudio sobre el uso de los antibióticos en España» ${ }^{7}$, las infecciones respiratorias bajas parecen ser más frecuentes en el ámbito rural $(30,4 \%)$ que en el urbano (22\%), invirtiéndose los términos en lo que respecta a las infecciones de oído/senos paranasales (16,2\% en el ámbito urbano y 7,6\% en el rural).

Rev Esp Salud Pública 2002, Vol. 76, N. ${ }^{\circ} 4$ 
Las diferencias de consumo de antibióticos entre diversas áreas geográficas están bien establecidas a través del estudio comparativo de los variados trabajos realizados en la geografía española ${ }^{8-10}$, aunque presenta el problema de contrastar metodologías diferentes (se objetivan distintas fuentes de información, indicadores, población asignada, etc.) y tiempos distintos (la variación que se produce a lo largo de los años está fuertemente contrastada), siendo escasos los trabajos con datos paralelos de consumo en varias áreas de una misma zona geográfica ${ }^{11}$. En el presente estudio tuvimos la ocasión de comparar el consumo de antibióticos de uso sistémico en la provincia de Valladolid dividida en seis áreas con las únicas variables $a$ priori de zona rural o urbana y la edad poblacional y los índices de dependencia de acuerdo a los resultados del estudio de la población.

Pudimos observar importantes desviaciones de consumo respecto a la media para el total de los cinco años del estudio, sin poder relacionar estas diferencias con los distintos índices demográficos de cada área. Un hecho que refuerza este punto es que la máxima diferencia de consumo se produjo entre Medina del Campo y el Área Centro, que sin embargo mostraron una distribución por edades similar. Asimismo las dos áreas con mayor diferencia de índices pediátrico y mayores de 65 años (Laguna de Duero y el Área Norte) mantuvieron consumos globales muy aproximados.

Por otra parte objetivamos que el consumo fue mayor en las áreas de carácter urbano (Medina del Campo, Valladolid capital y Laguna de Duero por este orden) respecto de las áreas de carácter rural. Otros autores $^{12,13}$ encuentran también la falta de asociación del consumo de antibióticos con la edad en mayores de 65 años pero asocian el entorno rural con un mayor grado de consumo, pudiendo deberse esta diferencia de resultados al uso de datos de prescripción y no de consumo real.
Estos datos sugieren la existencia de una variación entre médicos trabajando en áreas geográficas diferentes y por variaciones extremas dentro de un área geográfica, causadas por diferencias epidemiológicas en las infecciones, recursos médicos, así como la percepción individual de riesgo ${ }^{14}$ y los beneficios del tratamiento mantenido por médico y paciente, y la dinámica que afecte la relación entre ambos ${ }^{15}$.

\section{BIBLIOGRAFÍA}

1. Calvo Plaza MI, Moreno Úbeda R, Domínguez Rojas V, Prieto Prieto J. Cinco años de evolución del consumo extrahospitalario de betalactámicos en España (1993-1997). Rev Esp Quimioter 2000; 13: 417-24.

2. Lázaro Bengoa E, Madurga Sanz M, Abajo Iglesias FJ. Evolución del consumo de antibióticos en España, 1985-2000. Med Clin (Barc) 2002; 118 (15): $561-8$

3. Sociedad Española de Quimioterapia. Evaluación del consumo de antibióticos en la atención primaria de salud (1999). Rev Esp Quimioterap, Marzo 2001; Vol 14 (1): 69-72.

4. Base de Datos Municipales de Castilla y León: http://www.jcyl.es/jcyl/ceh/dge/

5. Martín Arias LH. Estudios de utilización de medicamentos. En: Carvajal A. Farmacoepidemiología. Valladolid: Universidad de Valladolid;1993; 37-48.

6. Pérez Gorricho B, Baquero F. Estrategias de uso y consumo de antibióticos. Med Clin (Barc) 1986; 86: 547-549.

7. Sociedad Española de Quimioterapia. Estudio sobre el uso de los antibióticos en España. Madrid: Gabinete Pfizer de Recursos Humanos; 1994.

8. Ruiz Bremón A, Ruíz-Tovar M, Pérez Gorricho B, Díaz de Torres P, López Rodríguez R. Non-hospital consumption of antibiotics in Spain: 1987-1997. J Antimicrob Chemother 2000 Mar; 45: 395-400.

9. García Lirola MA, Cabeza Barrera J, Ignacio García JM, Rabadán Asensio A. La calidad de la prescripción de antibacterianos en un distrito de atención primaria. Evolución 1994-1995. Aten Primaria 1997; 19: 487-492. 
10. Goldaracena Tanco M, Hernández Dettoma JL, Obaldía Alaña C, Ortega Martínez J, Siles Gutiérrez M. Utilización de antiinfecciosos en atención primaria en La Rioja. Aten Primaria 1998; 22: 575-579.

11. García Ruiz AJ, Fajardo Martín E, González Correa JA, Pavía Molina J, Sánchez de la Cuesta F. Utilización de antibacterianos en atención primaria en 1992. Farm Clín 1994; 11: 393-8.

12. Torralba Guirao M, Calero García MI, Segú i Tolsà JL, Faixedas Brunsoms MT, López Calahorra P. Factores que influyen sobre la utilización de antibióticos en atención primaria. Aten Primaria 1999; 24: 274-80.
13. Gonzales R, Steiner JF, Sade MA. Antibiotic prescribing for adults with colds, upper respiratory tract infections, and bronchitis by ambulatory care physicians. JAMA 1997; 278: 901-4.

14. Rotaeche del Campo R, Vicente Anza D, Mozo Avellaned $\mathrm{C}$ et al. Idoneidad de la prescripción antibiótica en atención primaria en la Comunidad Autónoma Vasca. Aten Primaria 2001; 27(9): 642-8.

15. Avorn J, Solomon DH. Cultural and economic factors that (mis)shape antibiotic use: thenonpharmacologic basis of therapeutics. Ann Intern Med 2000 Jul 18;133(2):128-35. 